\title{
Neuropsychiatric Aspects of Parkinson's Disease
}

Anna Nagy, MRCP, and Anette Schrag, FRCP, PhD

Department of Clinical and Movement Neurosciences, UCL Institute of Neurology, University College London, UK

Keywords: Neuropsychiatric symptoms; non-motor symptoms; Parkinson's disease; depression; anxiety; apathy; impulse control disorders; psychosis

Correspondence to:

Prof. Anette Schrag

Department of Clinical and Movement Neurosciences

UCL Institute of Neurology

University College London

NW3 2PF

Words: Abstract: 161, Article: 3453 


\section{Abstract}

Throughout the natural history of Parkinson's Disease (PD), from the pre-diagnostic phase to end stage disease, neuropsychiatric manifestations represent a core feature of the disease. This review summarises current knowledge on the features of depression, anxiety, apathy, fatigue, impulse control disorders (ICDs) and psychosis in PD. These co-morbidities are common and still frequently under-recognised. Their impact on quality of life is considerable, with depression having been shown to be the main determinant of health-related quality of life in PD patients. As well as formal diagnostic criteria, multiple scales have been developed to allow for assessment of these symptoms in both the clinic and research settings. The evidence base for treatment is variable, with increasing evidence for pharmacological and non-pharmacological treatment options for depression, but less robust evidence for the treatment of other neuropsychiatric features. Imaging studies are increasingly providing insight into the neural basis of these symptoms, with the hope that this insight will translate into treatments that can benefit patients. 


\section{Introduction}

Parkinson's disease (PD) is a classic neuropsychiatric condition, with multiple neuropsychiatric features occurring as presentations of the disease. Whilst more common in the advanced stages of these disease, and often related to antiparkinsonian medications, they can be present in the early stages and some even predate the diagnosis. In this review paper we outline common neuropsychiatric comorbidities in PD, risk factors, pathophysiology and treatment approaches.

\section{Depression}

\section{Prevalence and impact}

Depression is one of the main determinants of health-related quality of life in $\mathrm{PD}^{1}$ and associated with worse disability and prognosis. Depression is present at higher rates in PD patients compared to the age-matched healthy population and at higher rates than in other chronic diseases ${ }^{2}$. Diagnosis of depression is even significantly more common in PD patients than control subjects at least 5 years before diagnosis ${ }^{3}$ and physician-diagnosed depression is associated with an increased risk of PD with an odds ratio of $1 \cdot 6-2 \cdot 7^{4}$. Following diagnosis of PD, a wide range of prevalence rates for depression are reported, from $2.7 \%$ to $90 \%{ }^{5}$. In meta-analysis, clinically significant depression symptoms are present in $35 \%$ of patients and major depressive disorder in $17 \%$.

\section{Assessment}

Diagnosis of major depressive disorder in PD is based on the Diagnostic and Statistical Manual of Mental Disorders (DSM)-5 criteria requiring five or more symptoms during a two-week period, which represent a change from previous function. At least one of the symptoms must be either depressed mood or loss of interest or pleasure. However, it has been reported that depression may manifest differently in PD patients with less feelings of guilt, self-hate or lack of libido ${ }^{7}$. In addition, there is considerable overlap in symptoms between depression and other features of PD, leading to difficulties in accurate diagnosis.

Revised criteria for PD have therefore been proposed, which recommend that subsydromal depression in PD is recognised in patients who have depressive symptoms that do not meet criteria for diagnosis but have clinical relevance ${ }^{8}$. Validated rating scales for screening and severity ratings of depression in PD include the Beck Depression Inventory (BDI), Geriatric Depression Scale (GDS), 
Hospital Anxiety and Depression Scale (HADS-D), Hamilton Rating Scale for Depression (HAMD-17), Inventory of Depressive Symptomatology (IDS-C), Montgomery and Asberg Depression Rating Scale (MADRS), Patient Health Questionnaire (PHQ-9), World Health Organisation-Five Well-being Index (WHO-5) and Zun's Self-rating depression scale. These scales all have satisfactory screening properties with sensitivity $>85 \%{ }^{9}$.

\section{Treatment}

Treatment approaches should be tailored to the individual and guided by factors including comorbidities, other medications, the severity of the depressive symptoms and previous response to treatment as well as likelihood of adherence to treatment ${ }^{10}$. Depression can be related to motor fluctuations; approximately $75 \%$ of patients with motor fluctuations also experience prominent off period-related depressive symptoms ${ }^{11}$. If symptoms are associated with wearing off of levodopa therapy or undertreatment the first step is to optimise dopaminergic medications.

In depression unrelated to fluctuations in response to antiparkinsonian medication, nonpharmacological options should be considered first, with good evidence for the effectiveness of Cognitive Behavioural Therapy (CBT) in Randomised Controlled Trial (RCT) ${ }^{12}$. Pharmacological options include optimisation of antiparkinsonian medication including dopamine agonists and Monoamine Oxidase-B (MAO-B) inhibitors with the best trial evidence for improvement of depression in patients treated with pramipexole ${ }^{13}$. If additional antidepressant treatment is required, small RCTs support the use of tricyclic antidepressants, Selective Serotonin Reuptake Inhibitors, Serotonin and Norepinephrine reuptake inhibitors [summaries in ${ }^{5}$ and 14 ]. Antidepressant medication use should always consider interactions with or potentiation of side effects of concomitant medications (e.g. increasing risk of serotonin syndrome), and potential for a synergistic increase in other features of PD (e.g. increasing postural hypotension).

\section{Associations and new findings}

Whilst the relationship between motor severity and depression is only moderate, de novo patients who scored highly on the BDI showed more severe motor deficits and lower cognitive function ${ }^{15}$. Specifically, depression has been reported to be related to axial motor symptoms ${ }^{16}$. Imaging findings support the link with dopaminergic dysfunction, and PD patients with depression have lower striatal dopamine transporter binding than non-depressed patients in some studies ${ }^{17}$. However, other neurotransmitter systems are also affected with evidence for both serotonergic and noradrenergic dysfunction ${ }^{18}$. 
The neural correlates of depression in PD are likely to be diffuse. Imaging studies highlight the importance of the frontal lobe, with structural imaging showing more gray matter volume loss in the orbitofrontal area in PD patients with depression compared to those without ${ }^{19}$ and functional imaging showing lower regional brain perfusion in depressed PD patients in frontal gyri ${ }^{20}$.

However, there is good evidence for disrupted connectively extending to the temporal and occipital regions. In early PD patients there is impaired global efficiency and path length in those with depression, with disrupted connectivity in the left limbic and occipital regions ${ }^{21}$. Changes seen in imaging often appear to be asymmetric; diffusion MRI connectometry has also shown left sided changes with significant negative associations between quantitative anisotropy in the left cingulum, genu and splenium of corpus collosum. However, a recent study also highlighted a possible role for white matter pathways in the anterior and posterior limbs of the right internal capsule 22 .

Depressive symptoms also show links to other non-motor features, for example, a decrease in colour vision but not olfactory function has been reported to be associated with the severity of depression symptoms in PD patients ${ }^{23}$. Of particular interest, there is a complex relationship with cognitive function with bidirectional implications; depression can lead to worsening cognitive performance but depressive symptoms are also a risk factor for cognitive decline. In terms of cognitive profile, depression is most consistently associated with poor executive function and delayed episodic memory ${ }^{24}$.

\section{Anxiety}

\section{Prevalence and impact}

Anxiety is also an important neuropsychiatric comorbidity in PD patients with significant impact on quality of life ${ }^{25}$. Anxiety is often comorbid with depression. Some studies report that $70 \%$ of anxious patients in PD are also depressed ${ }^{26}$. Prevalence estimates range from $10 \%{ }^{27}$ to $55 \%{ }^{28}$. A systematic review found that $31 \%$ PD patients fulfil criteria for multiple anxiety disorders with clinically significant anxiety symptoms in $25.7 \%{ }^{29}$. Anxiety is also seen in the years before diagnosis of PD; anxious personality is associated with increased risk of PD with a hazard ratio of $1.63^{30}$. 


\section{Assessment}

Anxiety disorders are formally diagnosed according to DSM-5 criteria although this may not be entirely adequate for PD-related PD. A diagnosis of generalised anxiety disorder requires excessive anxiety and worry occurring more days than not for at least 6 months, and difficulty controlling the worry and associated symptoms. To reach criteria this must cause clinically significant distress or impairment in function. Rating scales are also important for assessment and monitoring, although the Beck Anxiety Inventory (BAI), Hospital Anxiety and Depression Scale (HADS) and the Hamilton Anxiety Rating Scale (HARS) have been validated for use in PD. The Parkinson Anxiety Scale (PAS) was designed as a PD-specific anxiety scale with high specificity and sensitivity ${ }^{31}$.

\section{Treatment}

There is little trial evidence for treatment of anxiety in $\mathrm{PD}^{32}$. Small open pilot studies suggest that $\mathrm{CBT}$, the standard treatment for anxiety disorders, may be of benefit. With regards to pharmacological treatments, empirically, off-period related anxiety is best treated with optimisation of antiparkinsonian medication, and standard pharmacological and non-pharmacological treatments for anxiety should be considered with appropriate consideration of possible side effects in this population.

\section{Associations and new findings}

Anxiety has been associated with younger age of onset and motor fluctuations ${ }^{33}$. It is also frequently co-morbid with depression, with the majority of anxious patients also experiencing depressive symptoms ${ }^{34}$. Risk factors for development of anxiety in PD include female gender, cognitive impairment, depressive symptoms, dysautonomia, insomnia and excessive daytime sleepiness $^{26}$.

Anxiety trait but not severity has been associated with left-sided amygdala atrophy ${ }^{35}$. An association between dopaminergic dysfunction and anxiety in do novo patients is also reported ${ }^{36}$.

\section{Apathy}




\section{Prevalence and Impact}

Apathy is increasingly recognised as a common neuropsychiatric syndrome common in neurodegenerative disease. The reported prevalence of apathy in PD ranges from $13.9 \%$ to $70 \%$ with a mean prevalence of $35 \%$ in patients overall. In studies where patients with comorbid depression and dementia are excluded, prevalence figures range from 3 to $47.9 \%{ }^{37}$.

\section{Assessment}

Proposed diagnostic criteria for apathy include both loss of motivation and presence of diminished goal directed behaviour and cognition as well as diminished concomitants of goal directed behaviour causing impairment or distress ${ }^{38}$. These were later modified by the European Alzheimer's Disease Consortium ${ }^{39}$ and have been validated in $\mathrm{PD}^{40}$. For screening and severity measurement, several questionnaires and rating scales have been developed and were evaluated by a task force commissioned by the Movement Disorders Society (MDS ${ }^{41}$; the only currently recommended scale for use in PD is the 14 item Apathy Scale ${ }^{42}$.

\section{Treatment}

There are no drugs approved for the treatment of apathy in PD. There is some evidence that, in non-demented and non-depressed apathetic PD patients, levodopa and dopamine agonists may improve subjective evaluation of motivation ${ }^{41,42}$. In addition, there is some evidence from small RCTs in non-demented patients with PD that rivastigmine ${ }^{43}$ and the selective D2/D3 dopamine agonist piribedil ${ }^{44}$ can improve apathy scores. Apathy after withdrawal from dopaminergic treatment in the context of deep brain stimulation of the subthalamic nucleus can respond to dopaminergic treatment ${ }^{45}$.

\section{Associations and new findings}

Apathy, along with depression, is implicated in poorer cognitive function in PD patients. There appears to be a summative effect of having both apathy and depression, with one study finding that patients reporting both disorders performed worse on tests of manual and psychomotor speech including verbal fluency than patients with either one or the other ${ }^{46}$. The underlying pathology is likely to be complex, involving dopaminergic as well as serotonergic dysfunction ${ }^{47}$, and potentially cholinergic and noradrenergic pathways ${ }^{48}$. Imaging studies have shown reduced grey matter density in the inferior frontal gyrus, insula and cingulate gyrus ${ }^{49}$. 


\section{Fatigue}

\section{Prevalence and Impact}

Fatigue is defined as an overwhelming sense of tiredness, lack of energy and feeling of exhaustion not attributable to drug side effects. In PD, fatigue is one of the most common and disabling nonmotor symptoms, affecting around $50 \%$ of patients in meta-analysis ${ }^{50}$. It is likely to be part of the pre-diagnostic phase of $\mathrm{PD}^{51}$ and persists over time during the disease course ${ }^{52}$. It leads to limitation of participation in both work and social activities, having a negative impact on quality of life ${ }^{53}$.

\section{Assessment}

The concept of pathological fatigue, involving feelings of tiredness at rest and lack of energy which compromises daily activities is variously defined in the literature. Jason et al suggest that to make a diagnosis of pathological fatigue, symptoms should be present for more than 3 months, be more intense than previously experienced fatigue and compromise daily activities and quality of life ${ }^{54}$. An MDS task force suggested proposed criteria for diagnosis of Parkinson's disease-related fatigue, requiring symptoms to be present most of the day every day or nearly every day during the past month, impairment in function, evidence that this is a consequence of PD and not co-morbid disorders or medical conditions ${ }^{55}$. Among rating scales also evaluated by the MDS task force, the fatigue severity scale (FSS) is the only scale recommended for both screening and grading the severity of fatigue. The multidimensional fatigue inventory is also recommended for grading the severity of fatigue and may be more sensitive than the FSS to change over time.

\section{Treatment}

An RCT comparing rasagiline to placebo for the treatment of fatigue reported that rasagiline improved scores on the modified fatigue impact scale in PD patients with moderate to severe fatigue ${ }^{56}$. The effects of dopaminergic treated on fatigue were also investigated in the ELLDOPA trial, where early PD patients were randomised to carbidopa-levodopa or placebo. In a secondary analysis, FSS scores increased in all treatment groups but there were no statistically significant differences at 40 weeks between the control and treatment groups. However, including a two-week washout period, there was a difference between groups, with a mean FSS worsening $19 \%$ in the placebo group compared to $7 \%$ and $8 \%$ in the levodopa groups ${ }^{57}$. A sub-study of the ADAGIO RCT also showed improvement in fatigue, with less progression of fatigue in the rasagiline arm than in the placebo arm over a 9 month period ${ }^{58}$. Modafinil, methylphenidate, tricyclic antidepressants, 
memantine and amantadine have not shown efficacy for fatigue in PD in RCTs ${ }^{59}$. Amongst nonpharmacological options acupuncture has been reported to show some benefit for fatigue in a small trial $^{60}$.

\section{Associations and new findings}

Sleep disorders, also common in PD, may confound the diagnosis of fatigue and PD patients with severe sleep disturbances tend to report higher levels of fatigue. Fatigue was shown in one study to be more prevalent in women ${ }^{53}$ but this association has not been borne out in meta-analysis. In a recent large meta-analysis, age, levodopa dosage, motor disability, cognitive function, depression, apathy, anxiety and sleep disorders were all associated with fatigue ${ }^{50}$. There is no difference in fatigue prevalence across levels of disease duration or in treated vs untreated patients suggesting that fatigue is present from early on in the disease.

\section{Impulse control disorders (ICDs) and Dopamine Dysregulation Syndrome (DDS)}

\section{Prevalence and Impact}

ICDs, resulting from dopaminergic treatment are now well recognised in PD patients. Dopamine agonists are particularly implicated in ICDs, increasing the odds of having an ICD by 2-3.5. There is comparable prevalence in pramipexole and ropinirole users. Use of levodopa, particularly at high dosages $^{61}$, and amantadine ${ }^{62}$ have also both been associated with ICDs. There are multiple personal implications of ICDs with financial, legal and medical complications as well as poorer quality of life and function ${ }^{63}$. ICDs occur in approximately 10 to $20 \%$ of PD patients over the course of the illness and can have substantial impact on both PD patients and their family and friends. Cross-sectional studies using formal assessments of ICDs report prevalence rates of $1.7-7 \%$ for compulsive gambling, $3.5 \%$ for compulsive sexual behaviour and $0.4-3 \%$ for compulsive buying. The DOMINION study using semi-structured interview and either rating scale or formal diagnostic criteria for the four main ICDs in medicated PD patients showed a prevalence $13.6 \%$ with $3.9 \%$ having two or more ICDs ${ }^{64}$. Risk factors for the ICDs in PD include male gender, younger age, a personal or family history of substance abuse and impulsive personality traits. Recently, smoking and increased caffeine have also been reported to be associated with ICDs in $\mathrm{PD}^{65}$. However, newly diagnosed patients do not show higher rates of ICDs than controls, suggesting that treatment is required for the development of these complications ${ }^{66}$. 


\section{Assessment}

ICD is classified under DSM-5 as a psychiatric disorder involving problems in the self-control of emotions and behaviours. In PD patients, four broad categories of ICD have been identified: pathological gambling, compulsive sexual behaviour, compulsive buying and binge-eating ${ }^{67}$, although other behaviours can also occur. Pathological gambling was previously included under the ICD classification in DSM-IV but in DSM-5 this have been re-classified under substance-related and addictive disorders and binge-eating is additionally included. DDS, a related impulsive behaviour, is described as an addiction-like state marked by self-medication with inappropriately high doses of high potency and short-acting medications, driven by a desire to maintain the "high" sensation of being in "on" state. The Questionnaire for Impulsive-Compulsive Disorders in Parkinson's DiseaseRating Scale (QUIP) is a self-completed screening questionnaire for ICDs and other compulsive behaviours and a short form QUIP-S has been recommended for routine use. The QUIP has excellent sensitivity (100\%) and sensitivity of $75 \%{ }^{68}$.

\section{Treatment}

Despite increasing recognition, there is evidence that ICD behaviours are still under-recognised and under-managed in clinical practice ${ }^{63}$. Both case reports and clinical experience suggest that ICDs resolve after reducing the dose of dopamine agonist, particularly with complete discontinuation of treatment. This approach is advocated by expert consensus, and class IV evidence ${ }^{69,70}$. However, this process can be complicated by dopamine agonist withdrawal syndrome, with features including anxiety, panic attacks, dysphoria, dysautonomia, sleep disturbance and cravings. This is little evidence for either drug or behavioural treatments. Naltrexone in a small RCT in PD showed benefit on self-reported scales but not clinician rating of change ${ }^{71}$. A small RCT of CBT showed benefit in global symptom severity ${ }^{72}$. Deep brain stimulation to the subthalamic nucleus has been reported as being effective in improving ICDs in a non-controlled case series ${ }^{73}$.

\section{Associations and new findings}

ICDs have been reported to be associated with worse cognitive performance: set-shifting and reward-related decision making, as well as depression, anxiety, anhedonia have all been associated with $\mathrm{ICDs}^{74}$. Rapid Eye Movement Sleep Behaviour Disorder (RBD) independently increases risk of $I C D s^{75}$. A number of genetic risk factors have been reported to be associated with ICDs in PD including the Parkin mutation, DRD2 polymorphism, GRIN2B and opioid receptor kappa. The pathophysiology of this vulnerability is suggested to be linked to abnormalities in dopaminergic binding. A positron emisson tomography study comparing dopaminergic function during gambling in 
PD patients with and without pathological gambling showed greater decreases in binding potential in the ventral striatum in those with pathological gambling ${ }^{76}$.

\section{Psychosis}

\section{Prevalence and impacts}

Psychosis is a common co-morbidity in PD with multiple manifestations. The prevalence of formed visual hallucinations has been estimated at $22-38 \%$, minor psychotic symptoms at $17-72 \%$, auditory hallucinations $0-22 \%$ and delusions $1-7 \%{ }^{77}$. The impact of psychosis is significant with increased caregiver burden, nursing home placement and mortality ${ }^{78}$. Visual hallucinations are particularly common and specific for Lewy body disorders, occurring in 50\% of PD patients, $73 \%$ of DLB patients and only $7 \%$ of patients with non-Lewy body parkinsonism, resulting in a specificity of $92.9 \%$ and a positive predictive value for $\mathrm{PD}$ of $93.4 \%{ }^{79}$.

\section{Assessment}

Diagnostic criteria for psychosis in PD have been proposed by a National Institute for Neurological Disorders and Stroke (NINDS)/National Institute of Mental Health (NIMH) working group ${ }^{80}$ requiring the presence of at least one of illusions, false sense of presence, hallucinations or delusions; primary diagnosis based on UK brain bank criteria for PD; symptoms occurring after the onset of PD; symptoms recurrent or continuous for one month and not better accounted for by another disorder such as dementia with Lewy bodies.

\section{Treatment}

Pimavanserin, a serotonin inverse agonist, has RCT evidence of efficacy in treating hallucinations and delusions compared to placebo in patients with PD psychosis without significant safety concerns at least in short-term follow-up ${ }^{81}$. It now has US Food and Drug Administration approval for the treatment of psychosis in PD. In several systematic reviews clozapine at comparatively low doses has been found to have good evidence for efficacy with improvement in clinical global impression. However, given the significant side effect profile of clozapine, regular monitoring is required and treatment must be balanced against risk and side effects. Quetiapine was not shown to be effective for psychosis in PD in placebo-controlled RCTs. It is nevertheless frequently used due to its tolerability and ease of use and support from several open label trials. Treatment with other typical and many atypical neuroleptics is contraindicated in Lewy body disorders due to the risk of 
potentially catastrophic motor deterioration. The acetylcholinesterase inhibitor rivastigmine, indicated for cognitive symptoms in PD, appears to be particularly beneficial in PD patients with visual hallucinations, who show larger improvements in cognition compared to non-hallucinators in placebo-controlled trials ${ }^{82}$.

Clonazepam was effective in reducing frequency of visual hallucinations in a small series of RBD patients ${ }^{83}$. Another small series of elderly PD patients showed improvement in psychiatric symptoms with electroconvulsive therapy ${ }^{84}$. In depression associated with mood congruent psychosis, antidepressant treatment with citalopram, venlafaxine and clomipramine alone can improve psychosis 85,86 .

\section{Associations and new findings}

Cognitive impairment has consistently been shown to be a risk factor for psychosis in PD, and drug treatment of cognitive features may improve psychotic features. The risk relationship is bidirectional, with visual hallucinations increasing risk of later dementia. Other risk factors include older age, longer disease duration, RBD, autonomic dysfunction, poor vision and dopaminergic medication. There is an increased risk of psychosis in PD with glucocerebrosidase (GBA) mutations ${ }^{87}$.

Imaging studies show atrophy in multiple areas in PD patients with psychosis, including superior parietal, lateral parietal, lateral and ventral occipitotemporal, lateral frontal and hippocampal as well as cerebellar. There is consistent evidence of posterior changes in metabolism and perfusion but the involvement of the occipital lobe remains unclear ${ }^{88}$. There is also evidence that Lewy body pathology plays a key role, with associations between temporal Lewy body density and the presence of visual hallucinations ${ }^{89}$.

\section{Conclusions}

Neuropsychiatric features are common and disabling features of PD. There is increasing understanding of their clinical characteristics and underlying pathophysiology but treatment response to medication may be poor and interventions are often insufficiently tested. The underlying pathology of neuropsychiatric features in PD and the side effects of classical treatments can limit their usefulness in patients with PD. Whilst significant progress has been made to explore 
depression in PD and its treatment, other neuropsychiatric features such as anxiety, apathy and ICDs are still poorly understood and remain difficult to treat. Future research in these areas will improve the basis and evidence for targeted treatment of these important aspects of PD.

\section{References}

1. Schrag, A. Quality of life and depression in Parkinson's disease. J. Neurol. Sci. 248, 151-157 (2006).

2. Nilsson, F. M., Kessing, L. V., Sørensen, T. M., Andersen, P. K. \& Bolwig, T. G. Major depressive disorder in Parkinson's disease: a register-based study. Acta Psychiatr Scand 106, 202-211 (2002).

3. Schrag, A., Horsfall, L., Walters, K., Noyce, A. \& Petersen, I. Prediagnostic presentations of Parkinson's disease in primary care: a case-control study. Lancet Neurol 14, 57-64 (2015).

4. Fang, F. et al. Depression and the subsequent risk of Parkinson's disease in the NIH-AARP Diet and Health Study. Mov. Disord. 25, 1157-1162 (2010).

5. Timmer, M. H. M., van Beek, M. H. C. T., Bloem, B. R. \& Esselink, R. A. J. What a neurologist should know about depression in Parkinson's disease. Pract Neurol 17, 359-368 (2017).

6. Reijnders, J. S. A. M., Ehrt, U., Weber, W. E. J., Aarsland, D. \& Leentjens, A. F. G. A systematic review of prevalence studies of depression in Parkinson's disease. Mov. Disord. 23, 183-9quiz 313 (2008).

7. Kritzinger, C. et al. Qualitative Characteristics of Depression in Parkinson's Patients and Controls. Behav Neurol 2015, 961372-5 (2015).

8. Marsh, L., McDonald, W. M., Cummings, J., Ravina, B.NINDS/NIMH Work Group on Depression and Parkinson's Disease. Provisional diagnostic criteria for depression in Parkinson's disease: report of an NINDS/NIMH Work Group. Mov. Disord. 21, 148-158 (2006).

9. Martinez-Martin, P. et al. Accuracy of screening instruments for detection of neuropsychiatric syndromes in Parkinson's disease. Mov. Disord. 31, 270-279 (2016).

10. Pilling, S. et al. Depression in adults, including those with a chronic physical health problem: summary of NICE guidance. BMJ 339, b4108-b4108 (2009).

11. Richard, I. H., Justus, A. W. \& Kurlan, R. Relationship between mood and motor fluctuations in Parkinson's disease. J Neuropsychiatry Clin Neurosci 13, 35-41 (2001).

12. Egan, S. J., Laidlaw, K. \& Starkstein, S. Cognitive Behaviour Therapy for Depression and Anxiety in Parkinson's Disease. J Parkinsons Dis 5, 443-451 (2015).

13. Barone, P. et al. Pramipexole for the treatment of depressive symptoms in patients with Parkinson's disease: a randomised, double-blind, placebo-controlled trial. Lancet Neuro/ 9, 573-580 (2010).

14. Seppi, K. et al. Update on treatments for nonmotor symptoms of Parkinson's disease-an evidence-based medicine review. Mov. Disord. 17, S1 (2019).

15. Lee, Y. et al. The presence of depression in de novo Parkinson's disease reflects poor motor compensation. PLOS ONE 13, e0203303 (2018).

16. Brown, R. G. et al. Depression and anxiety related subtypes in Parkinson's disease. J. Neurol. Neurosurg. Psychiatr. 82, 803-809 (2011).

17. Vriend, C. et al. Depression and impulse control disorders in Parkinson's disease: two sides of the same coin? Neurosci Biobehav Rev 38, 60-71 (2014).

18. Gallagher, D. A. \& Schrag, A. Psychosis, apathy, depression and anxiety in Parkinson's disease. Neurobiol. Dis. 46, 581-589 (2012).

19. Feldmann, A. et al. Morphometric changes of gray matter in Parkinson's disease with depression: a voxel-based morphometry study. Mov. Disord. 23, 42-46 (2008). 
20. Mayberg, H. S. et al. Selective hypometabolism in the inferior frontal lobe in depressed patients with Parkinson's disease. Annals of Neurology 28, 57-64 (1990).

21. Gou, L. et al. Structural Brain Network Alteration and its Correlation With Structural Impairments in Patients With Depression in de novo and Drug-Naïve Parkinson's Disease. Front Neurol 9, 608 (2018).

22. Ghazi Sherbaf, F., Same, K. \& Aarabi, M. H. High angular resolution diffusion imaging correlates of depression in Parkinson's disease: a connectometry study. Acta Neurol Belg 118, 573-579 (2018).

23. Li, D.-K. et al. Depressive Symptoms Are Associated With Color Vision but not Olfactory Function in Patients With Parkinson's Disease. J Neuropsychiatry Clin Neurosci 30, 122-129 (2018).

24. Poletti, M., De Rosa, A. \& Bonuccelli, U. Affective symptoms and cognitive functions in Parkinson's disease. J. Neurol. Sci. 317, 97-102 (2012).

25. Qureshi, S. U., Amspoker, A. B., Calleo, J. S., Kunik, M. E. \& Marsh, L. Anxiety disorders, physical illnesses, and health care utilization in older male veterans with Parkinson disease and comorbid depression. J Geriatr Psychiatry Neurol 25, 233-239 (2012).

26. Zhu, K., van Hilten, J. J. \& Marinus, J. Onset and evolution of anxiety in Parkinson's disease. Eur. J. Neurol. 24, 404-411 (2017).

27. Mondolo, F. et al. Evaluation of anxiety in Parkinson's disease with some commonly used rating scales. Neurol. Sci. 28, 270-275 (2007).

28. Quelhas, R. \& Costa, M. Anxiety, depression, and quality of life in Parkinson's disease. J Neuropsychiatry Clin Neurosci 21, 413-419 (2009).

29. Broen, M. P. G., Narayen, N. E., Kuijf, M. L., Dissanayaka, N. N. W. \& Leentjens, A. F. G. Prevalence of anxiety in Parkinson's disease: A systematic review and meta-analysis. Mov. Disord. 31, 1125-1133 (2016).

30. Bower, J. H. et al. Anxious personality predicts an increased risk of Parkinson's disease. Mov. Disord. 25, 2105-2113 (2010).

31. Forjaz, M. J. et al. Is the Parkinson Anxiety Scale comparable across raters? Mov. Disord. 30, 545-551 (2015).

32. Seppi, K. et al. The Movement Disorder Society Evidence-Based Medicine Review Update: Treatments for the non-motor symptoms of Parkinson's disease. Mov. Disord. 26 Suppl 3, S4280 (2011).

33. Burn, D. J. et al. Parkinson's disease motor subtypes and mood. Mov. Disord. 27, 379-386 (2012).

34. Starkstein, S. E., Robinson, R. G., Leiguarda, R. \& Preziosi, T. J. Anxiety and depression in Parkinson's disease. Behav Neurol 6, 151-154 (1993).

35. Vriend, C. et al. A smaller amygdala is associated with anxiety in Parkinson's disease: a combined FreeSurfer-VBM study. J. Neurol. Neurosurg. Psychiatr. 87, 493-500 (2016).

36. Picillo, M. et al. Association between dopaminergic dysfunction and anxiety in de novo Parkinson's disease. Parkinsonism \& Related Disorders 37, 106-110 (2017).

37. Santangelo, G. et al. Apathy in Parkinson's disease: diagnosis, neuropsychological correlates, pathophysiology and treatment. Behav Neurol 27, 501-513 (2013).

38. Starkstein, S. E. \& Leentjens, A. F. G. The nosological position of apathy in clinical practice. J. Neurol. Neurosurg. Psychiatr. 79, 1088-1092 (2008).

39. Robert, P. et al. Proposed diagnostic criteria for apathy in Alzheimer's disease and other neuropsychiatric disorders. Eur. Psychiatry 24, 98-104 (2009).

40. Drijgers, R. L., Dujardin, K., Reijnders, J. S. A. M., Defebvre, L. \& Leentjens, A. F. G. Validation of diagnostic criteria for apathy in Parkinson's disease. Parkinsonism \& Related Disorders 16, 656660 (2010).

41. Leentjens, A. F. G. et al. Apathy and anhedonia rating scales in Parkinson's disease: Critique and recommendations. Mov. Disord. 23, 2004-2014 (2008). 
42. Starkstein, S. E. et al. Reliability, validity, and clinical correlates of apathy in Parkinson's disease. J Neuropsychiatry Clin Neurosci 4, 134-139 (1992).

43. Devos, D. et al. Rivastigmine in apathetic but dementia and depression-free patients with Parkinson's disease: a double-blind, placebo-controlled, randomised clinical trial. J. Neurol. Neurosurg. Psychiatr. 85, 668-674 (2014).

44. Thobois, S. et al. Parkinsonian apathy responds to dopaminergic stimulation of D2/D3 receptors with piribedil. Brain 136, 1568-1577 (2013).

45. Czernecki, V. et al. Apathy following subthalamic stimulation in Parkinson disease: A dopamine responsive symptom. Mov. Disord. 23, 964-969 (2008).

46. Cohen, M. L., Aita, S., Mari, Z. \& Brandt, J. The Unique and Combined Effects of Apathy and Depression on Cognition in Parkinson's Disease. J Parkinsons Dis 5, 351-359 (2015).

47. Maillet, A. et al. The prominent role of serotonergic degeneration in apathy, anxiety and depression in de novo Parkinson's disease. Brain 139, 2486-2502 (2016).

48. Schrag, A. \& Politis, M. Serotonergic loss underlying apathy in Parkinson's disease. Brain 139, 2338-2339 (2016).

49. Reijnders, J. S. A. M. et al. Neuroanatomical correlates of apathy in Parkinson's disease: A magnetic resonance imaging study using voxel-based morphometry. Mov. Disord. 25, 23182325 (2010).

50. Siciliano, M. et al. Fatigue in Parkinson's disease: A systematic review and meta-analysis. Mov. Disord. 2, 15025 (2018).

51. Pont-Sunyer, C. et al. The onset of nonmotor symptoms in Parkinson's disease (the ONSET PD study). Mov. Disord. 30, 229-237 (2015).

52. Friedman, J. H. \& Friedman, H. Fatigue in Parkinson's disease: a nine-year follow-up. Mov. Disord. 16, 1120-1122 (2001).

53. Barone, P. et al. The PRIAMO study: A multicenter assessment of nonmotor symptoms and their impact on quality of life in Parkinson's disease. Mov. Disord. 24, 1641-1649 (2009).

54. Jason, L. A., Evans, M., Brown, M. \& Porter, N. What is fatigue? Pathological and nonpathological fatigue. PM R 2, 327-331 (2010).

55. Kluger, B. M. et al. Parkinson's disease-related fatigue: A case definition and recommendations for clinical research. Mov. Disord. 31, 625-631 (2016).

56. Lim, T. T. et al. Rasagiline for the symptomatic treatment of fatigue in Parkinson's disease. Mov. Disord. 30, 1825-1830 (2015).

57. Schifitto, G. et al. Fatigue in levodopa-naive subjects with Parkinson disease. Neurology 71, 481-485 (2008).

58. Stocchi, F.ADAGIO investigators. Benefits of treatment with rasagiline for fatigue symptoms in patients with early Parkinson's disease. Eur. J. Neurol. 21, 357-360 (2014).

59. Nassif, D. V. \& Pereira, J. S. Fatigue in Parkinson's disease: concepts and clinical approach. Psychogeriatrics 18, 143-150 (2018).

60. Corbin, L. et al. Acupuncture for Symptomatic Treatment of Fatigue in Parkinson's Disease: Trial Design and Implementation. Med Acupunct 28, 194-205 (2016).

61. Pontieri, F. E. et al. Sociodemographic, neuropsychiatric and cognitive characteristics of pathological gambling and impulse control disorders NOS in Parkinson's disease. Eur Neuropsychopharmacol 25, 69-76 (2015).

62. Weintraub, D. et al. Amantadine use associated with impulse control disorders in Parkinson disease in cross-sectional study. Annals of Neurology 68, 963-968 (2010).

63. Phu, A. L. et al. Effect of impulse control disorders on disability and quality of life in Parkinson's disease patients. J Clin Neurosci 21, 63-66 (2014).

64. Weintraub, D. et al. Impulse control disorders in Parkinson disease: a cross-sectional study of 3090 patients. Arch. Neurol. 67, 589-595 (2010).

65. Zurowski, M. \& O'Brien, J. D. Developments in impulse control behaviours of Parkinson's disease. Curr. Opin. Neurol. 28, 387-392 (2015). 
66. O'Callaghan, C. \& Hornberger, M. Screening for impulse control symptoms in patients with de novo Parkinson disease: a case-control study. Neurology 81, 694-695 (2013).

67. Weintraub, D. \& Claassen, D. O. Impulse Control and Related Disorders in Parkinson's Disease. Int. Rev. Neurobiol. 133, 679-717 (2017).

68. Papay, K. et al. Patient versus informant reporting of ICD symptoms in Parkinson's disease using the QUIP: validity and variability. Parkinsonism \& Related Disorders 17, 153-155 (2011).

69. Samuel, M. et al. Management of impulse control disorders in Parkinson's disease: Controversies and future approaches. Mov. Disord. 30, 150-159 (2015).

70. Lee, J.-Y. et al. Behavioural and trait changes in parkinsonian patients with impulse control disorder after switching from dopamine agonist to levodopa therapy: results of REIN-PD trial. J. Neurol. Neurosurg. Psychiatr. 90, 30-37 (2019).

71. Weintraub, D., Papay, K. \& Xie, S. X. Naltrexone for impulse control disorders in Parkinson disease: a placebo-controlled study. Neurology 84, 1386-1387 (2015).

72. Okai, D. et al. Trial of CBT for impulse control behaviors affecting Parkinson patients and their caregivers. Neurology 80, 792-799 (2013).

73. Abbes, M. et al. Subthalamic stimulation and neuropsychiatric symptoms in Parkinson's disease: results from a long-term follow-up cohort study. J. Neurol. Neurosurg. Psychiatr. 89, 836-843 (2018).

74. Martini, A., Dal Lago, D., Edelstyn, N. M. J., Grange, J. A. \& Tamburin, S. Impulse Control Disorder in Parkinson's Disease: A Meta-Analysis of Cognitive, Affective, and Motivational Correlates. Front Neurol 9, 654 (2018).

75. Fantini, M. L. et al. Sleep and REM sleep behaviour disorder in Parkinson's disease with impulse control disorder. J. Neurol. Neurosurg. Psychiatr. 89, 305-310 (2018).

76. Steeves, T. D. L. et al. Increased striatal dopamine release in Parkinsonian patients with pathological gambling: a [11C] raclopride PET study. Brain 132, 1376-1385 (2009).

77. Fénelon, G. \& Alves, G. Epidemiology of psychosis in Parkinson's disease. J. Neurol. Sci. 289, 12-17 (2010).

78. Goetz, C. G. \& Stebbins, G. T. Mortality and hallucinations in nursing home patients with advanced Parkinson's disease. Neurology 45, 669-671 (1995).

79. Williams, D. R. \& Lees, A. J. Visual hallucinations in the diagnosis of idiopathic Parkinson's disease: a retrospective autopsy study. Lancet Neurol 4, 605-610 (2005).

80. Ravina, B. et al. Diagnostic criteria for psychosis in Parkinson's disease: report of an NINDS, NIMH work group. Mov. Disord. 22, 1061-1068 (2007).

81. Cummings, J. et al. Pimavanserin for patients with Parkinson's disease psychosis: a randomised, placebo-controlled phase 3 trial. Lancet 383, 533-540 (2014).

82. Burn, D. et al. Effects of rivastigmine in patients with and without visual hallucinations in dementia associated with Parkinson's disease. Mov. Disord. 21, 1899-1907 (2006).

83. Nomura, T. et al. Visual hallucinations as REM sleep behavior disorders in patients with Parkinson's disease. Mov. Disord. 18, 812-817 (2003).

84. Ueda, S., Koyama, K. \& Okubo, Y. Marked improvement of psychotic symptoms after electroconvulsive therapy in Parkinson disease. J ECT 26, 111-115 (2010).

85. Voon, V., Fox, S., Butler, T. R. \& Lang, A. E. Antidepressants and psychosis in Parkinson disease: a case series. Int J Geriatr Psychiatry 22, 601-604 (2007).

86. Meco, G. \& Bernardi, S. Antidepressant use in treatment of psychosis with comorbid depression in Parkinson's disease. Prog. Neuropsychopharmacol. Biol. Psychiatry 31, 311-313 (2007).

87. Li, Y. et al. Clinicogenetic study of GBA mutations in patients with familial Parkinson's disease. Neurobiol. Aging 35, 935.e3-8 (2014).

88. ffytche, D. H. \& Aarsland, D. Psychosis in Parkinson's Disease. Int. Rev. Neurobiol. 133, 585-622 (2017). 
89. Harding, A. J., Broe, G. A. \& Halliday, G. M. Visual hallucinations in Lewy body disease relate to Lewy bodies in the temporal lobe. Brain 125, 391-403 (2002). 